\title{
Research of Structure and Characteristics for Tribological Electrostatic Sensors
}

\author{
Ruochen Liu*, Shaoyi Bei and Jing Zhou \\ Department of Traffic and Transportation, Jiangsu University of Technology, School of Automobile and Traffic Engineering, \\ No.1801 ZhongWu Avenue, Changzhou, 213001, China \\ ${ }^{*}$ Corresponding author
}

\begin{abstract}
A novel technique called electrostatic sensing method has been applied to condition monitoring on tribological system. Two basic types of electrostatic sensors are Wear-Site Sensor (WSS) and Oil-Line Sensor (OLS). According to the sensor structure (shape and size), different sensors have different features. This paper analyzed the characteristics of electrostatic sensors with modified standard mathematical model for each kinds. Useful conclusions were investigated and listed by contrast, lead to a better understanding and application for the technique.
\end{abstract}

Keywords—sensor structure; mathematical model; electrostatic sensing; WSS; OLS

\section{INTRODUCTION}

Electrostatic sensing is a novel technique based on electrostatic charging phenomena for condition monitoring [1]. In tribological system, there are two types of electrostatic sensors currently available to detect charge produced as a result of wear, seen in figure 1. Wear-site sensor (WSS) [2-4] with a flat sensing face that can be positioned directly above or near a wear surface. Oil-line sensor (OLS) [5-8] with a ring type sensing face that can be put in a lubricant flow downstream from wear components.

Although some investigations have been undertaken on the electrostatic monitoring techniques through experimental studies, few efforts have been made to investigate the influence of sensor structures and feature on the detection. Morris [9] used a number of charged steel balls with different diameters to investigate the sensor response and the best sensor geometry was selected, while Harvey [10] developed an oil droplet rig to investigate the electrostatic charges. The concept of the sensor efficiency and the field of view for OLS was firstly raised by Morris [9]. Gajewski and Yan et al. [11-14] established a mathematical model for the ring type probe based on charging principles, focused on the output type. Liu [15-17] further investigated the simulation and calibration method for both OLS and WSS. However no further investigations overall contrast the two type of electrostatic sensors for better understanding and optimization.

Based on previous researches, this paper combined the two type of electrostatic sensors to illustrate their own characteristics. Both electrostatic WSS and OLS were considered by detailed contrast and analysis. Important parameters were listed and the simulation and experimental results were concluded. It is a guidance for electrostatic sensors optimization with better industry application.

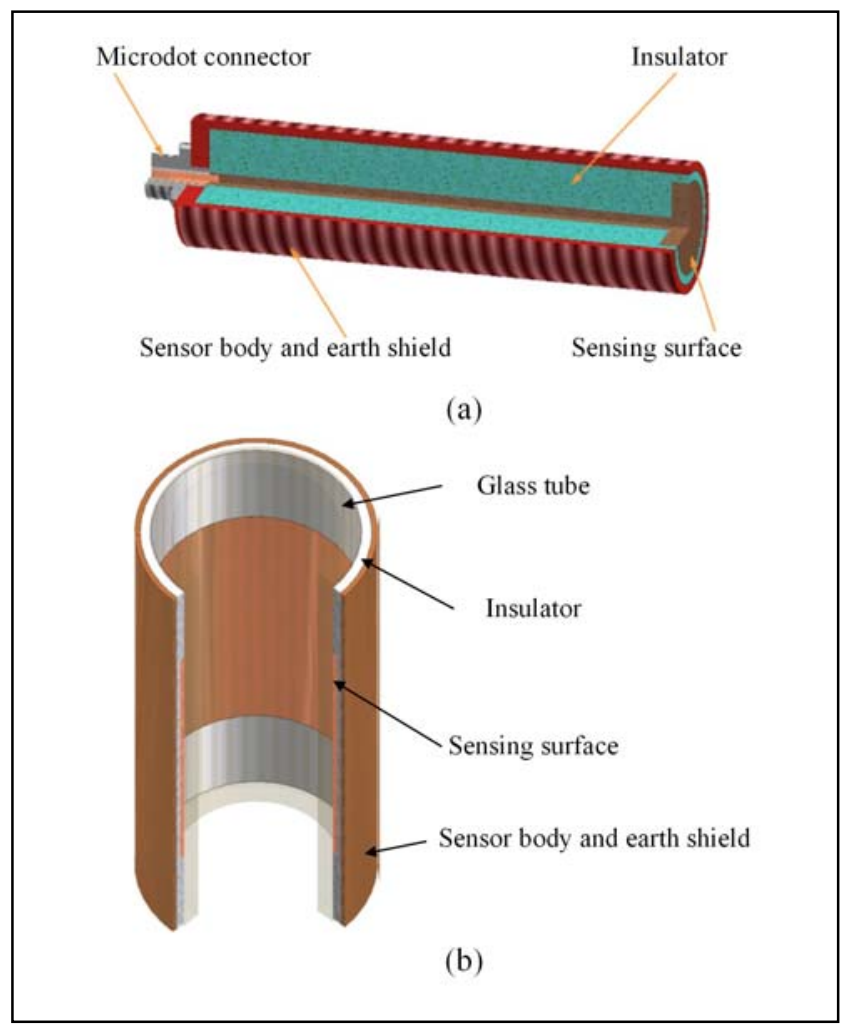

FIGURE I. STRUCTURE OF ELECTROSTATIC SENSORS (A) WSS (B) OLS

\section{Mathematical Model of Electrostatic SEnsor}

\section{A. Electrostatic Sensing Technique}

The principle of electrostatic monitoring is simple and easy to be explained. When a charge source, such as charged debris or lubricant charge species (e.g. additives or soot particles), or the combination of the two, passes through the bore of the sensor, an equal opposite charge will be induced on the sensing face that can be detected as a charge response. For multiple charge sources, the overall charge signal can be obtained by adding the single individual charge sources together. Therefore, single charge source is used and discussed in details during sensor simulation and validation using experimental data. 


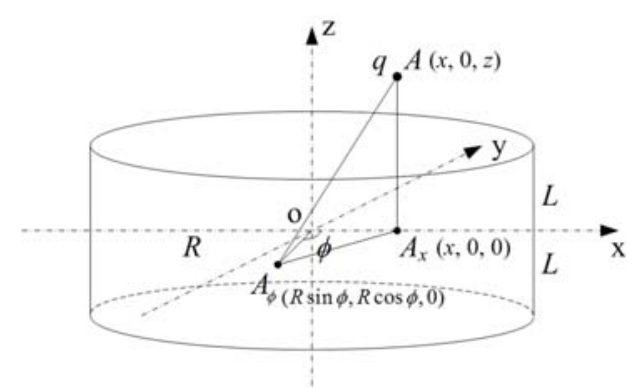

(a)

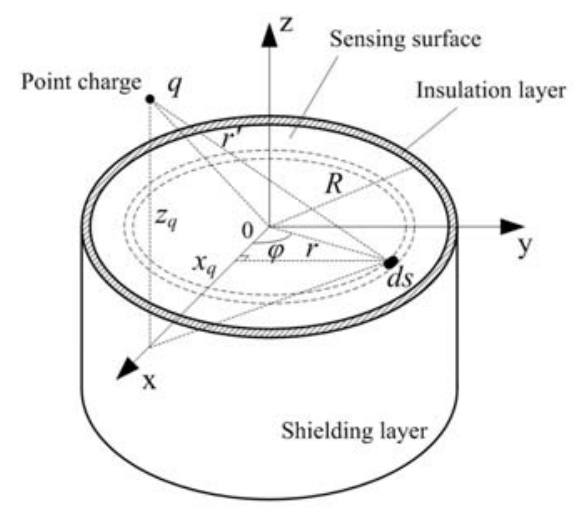

(b)

FIGURE II. MATHEMATICAL MODEL OF ELECTROSTATIC SENSORS (A) OLS (B) WSS

\section{B. Mathematical Model}

Although the principle of electrostatic monitoring sensors is the same, the mathematical model of OLS and WSS is different, considering their different shapes. Figure 2(a) shows the space coordinate system of a point charge in the sensing zone of an OLS according to the mathematical model provided in [17]. By Coulomb's law and Gauss theorem, using the notations in figure 2(a), the total induced charge $Q$ on the inner surface of the probe for a given point charge $q$ can be calculated using the following equation

$$
\begin{aligned}
& Q=\frac{R q}{2 \pi} \int_{0}^{\pi} \frac{R-x \cos \phi}{R^{2}+x^{2}-2 R x \cos \phi} \\
& \int \frac{z+L}{\sqrt{(z+L)^{2}+\left(R^{2}+x^{2}-2 R x \cos \phi\right)}}- \\
& \left.\frac{z-L}{\sqrt{(z-L)^{2}+\left(R^{2}+x^{2}-2 R x \cos \phi\right)}}\right) d \phi
\end{aligned}
$$

The meaning of variable and symbol in the equation is the same and detailed explained in [17].

While figure 2(b) shows the space coordinate system of a point charge in the sensing zone of a WSS according to the mathematical model provided in [15]. Equally by Coulomb's law and Gauss theorem, using the notations in figure 2(b), the total induced charge $Q$ on the inner surface of the probe for a given point charge $q$ can be calculated using the following equation

$$
Q=-\frac{q z}{4 \pi} \int_{0}^{R} \int_{0}^{2 \pi} \frac{r}{\left(z^{2}+r^{2}+x^{2}-2 r x \cos \varphi\right)^{\frac{3}{2}}} d \varphi d r
$$

The meaning of variable and symbol in the equation is the same and detailed explained in [15].

\section{CHARACTERISTIC PARAMETERS}

The performance of electrostatic sensors should be clearly presented by using useful natural parameters while monitoring. A series of parameters have been carried out to reflect the feature of both electrostatic WSS and OLS, such as spatial sensitivity, sensor efficiency, static sensitivity, field of view.

\section{A. Spatial Sensitivity}

The spatial sensitivity of electrostatic probe is defined as the negative value of the ratio of the induced charge to the inducing point charge and is represented by the following dimensionless parameter.

$$
s(x, z)=-Q / q(x, z)
$$

Where $s$ is the spatial sensitivity, $Q$ is the induced charge on the probe and $q$ is the inducing point charge, $x$ and $z$ are the space relative radial and axial position of the point charge to the probe. The induced charge $Q$ can be calculated using the above mentioned mathematical model for a point charge at any positions in the sensing zone.

\section{B. Sensor Efficiency}

Sensor efficiency or sensitivity is an important parameter that measures the sensor's detection ability. Since the spatial sensitivity and input-output correlation curve change with the position of the inducing point charge, a relatively static parameter i.e. sensor efficiency corresponding to a specific and fixed spatial position to the probe which can directly reflect the minimum detection ability while the inducing charges are passing through the sensing zone [9]. It means no matter how the charge sources pass through the sensor (position, velocity, trajectory and rotation), the detected output can at least have the corresponding ratio of values to the inducing charges.

Morris [9] defined the sensor efficiency as the ratio of the negative value of the induced charge on the probe to the inducing point charge at a specific position in the sensing zone of the OLS and multiply by $100 \%$. The theoretical efficiency can be thus be expressed by the following

$$
\eta=-Q / q\left(x_{0}, z_{0}\right) \times 100 \%
$$


Where $\eta$ is the sensor theoretical efficiency, $Q$ is the induced charge on the probe and $q$ is the inducing point charge, $x_{0}$ and $z_{0}$ are the selected specific space relative radial and axial position to the probe. It is obvious that the sensor theoretical efficiency is actually equal to the spatial sensitivity of the position $\left(x_{0}, z_{0}\right)$ to the probe.

\section{Static Sensitivity}

Different from the spatial sensitivity, the static sensitivity is defined as the ratio of the increment of voltage of the sensor output to the increment of charge of the sensor input, which can be expressed by the following

$$
s^{\prime}=\Delta U^{\prime} / \Delta q^{\prime}
$$

Where $s^{\prime}$ is sensor static sensitivity, $\Delta U^{\prime}$ is the increment of voltage of the sensor's output and $\Delta q^{\prime}$ is the increment of charge of the sensor's input. The corresponding position of this parameter will be further explained in the experimental setup and discussion below.

\section{Field of View}

Similar to that in optics [18-19], 'field of view' is introduced as an essential and significant parameter for electrostatic sensors due to the fact that sensors only detect charge in the field of the sensing zone of a probe. The electrostatic field of view was defined as the measurable maximum range in space detected by the electrostatic sensor when an inducing charge passes through the sensing zone of the sensor probe.

\section{RESUlTS OF SiMULATION AND EXPERIMENT}

The simulation for both electrostatic wear-site sensors (WSSs) and oil-line sensors (OLSs) have been calculated through their mathematical models. Then experiments have been taken to validate the results. The test rig and specific results can be searched and studied in [9-17]. By contrasting and analyzing these results, the main conclusions can be summarized below.

First, the spatial sensitivity of a WSS increases with the decrease of both axial position and radial position within in the corresponding sensing zone of the probe surface. The spatial sensitivity also exists outside the corresponding sensing zone of the probe surface and has the same trends in radial direction, but it first increases and then decreases in axial direction due to the angle and distance between the spatial position and probe surface. For the same spatial position, the spatial sensitivity increases with the increase of probe diameter. The static sensitivity further probes the variation trend through the inputoutput correlation curve with multiple tests. While the spatial sensitivity of an OLS increases with the decrease of axial position and the increase of radial position inside the probe. The spatial sensitivity also exists outside of the probe but has the opposite trends as those inside the probe.

Second, the ratio of the probe length and diameter is a significant parameter for electrostatic OLSs and the higher the ratio the higher the spatial sensitivity and theoretical efficiency can be achieved. Electrostatic OLSs with the same ratio have the same theoretical efficiency, even when the probe length and diameter are different. The real sensor efficiency obtained through experiment is in agreement with the theoretical efficiency, which successfully validated the simulated theoretical efficiency curve. While the ratio of probe-radius and centre-distance is a significant parameter for electrostatic WSSs which is related to the detection point in application. The higher the ratio, the higher the spatial sensitivity and theoretical efficiency can be achieve to the theoretical maximum of 0.5 .

Third, the electrostatic field of view calculated based on the measurements from a range of OLSs at different probe lengths and diameters shows that, the field of view increases with the increase of normalized radial position and has the maximum at the centre line of the sensor. However OLSs with larger probe length and diameter have a larger field of view, even when the ratios of the probe length and diameter are the same. While the electrostatic field of view calculated based on the measurements for WSSs shows that, the field of view increases with the increase of probe diameter. However the efficiencies at the edges are very low and nearly the same for these tested WSSs.

In summary, it is recommended that in the applications of electrostatic OLSs, sensors with larger ratio of the probe length and diameter should be firstly selected to increase the sensor efficiency with a given diameter of the oil pipe, while the overall size of the OLS should be increased as much as possible within the restriction of installation and fixation. While it is recommended that in the applications of electrostatic WSSs, sensors with larger sensing surface should be firstly selected to increase the sensor efficiency and sensitivity, meanwhile the distance between the probe and the detection point should be as much close as possible within the restriction of installation and fixation. Thus a comprehensive consideration should be researched to decide the most suitable size of the electrostatic sensor eventually for both OLS and WSS. It is more important to consider the actual industry environment where complex conditions may affect the performance of electrostatic both OLSs and WSSs such as strong background noises.

\section{CONCLUSIONS}

This paper has investigated the structure and characteristics for tribological electrostatic monitoring sensors. OLS and WSS, two typical types of electrostatic sensors, have been analyzed and compared to further explain about the simulation and experimental results. The listed parameters are useful for describing electrostatic sensor characteristics. The results show that electrostatic OLSs and WSSs have different change regulations with different sensor shapes and sizes. They can be further optimized for better industry application.

\section{ACKNOWLEDGMENT}

This project is supported by National Natural Science Foundation of China (Grant No.51705221) and the Program of Science and Technology Foundation of Changzhou (Grant No. CE20175027). The authors would like to thank Prof. Zuo H.F., Cai J. and Sun J.Z. of Nanjing University of Aeronautics and Astronautics for their valuable suggestions. 


\section{REFERENCES}

[1] R. C. Liu, H. F. Zuo, J. Z. Sun, and S. Y. Bei, "A Review on Electrostatic Monitoring”, 2017 International Conference on Sensing, Diagnostics, Prognostics, and Control, August, pp. 128-131, 2017.

[2] R. C. Liu, H. F. Zuo, J. Z. Sun, X. Li, and H. J. Mao, "Electrostatic monitoring of vehicle gearbox," Chinese Journal of Traffic and Transportation Engineering, vol. 15, no. 1, pp. 50-57,73, 2015.

[3] R. C. Liu, and H. F. Zuo, "Research on electrostatic monitoring method of rolling bearings with injected fault under variable operating conditions,” Chinese Journal of Scientific Instrument, vol. 35, no. 10, pp. 2348-2355, 2014.

[4] R. C. Liu, H. F. Zuo, Y. Zhang, and K. Liang, "Electrostatic monitoring of oil lubricated rolling bearing performance degradation and experiment,” Chinese Journal of Mechanical Engineering, vol. 50, no. 23, pp. 75-81, 2014.

[5] R. C. Liu, H. F. Zuo, H. J. M, and J. Z. Sun, "Simulation and experiment of dynamic characteristics for oil-line electrostatic sensor," Chinese Journal of Aerospace Power, vol. 32, no. 10, pp. 2438-2446, 2017.

[6] R. Hanzal, "Rolling contact fatigue failures in silicon nitride and their detection,” University of Southampton, 2013.

[7] M. Craig, T. J. Harvey, R. J. K. Wood, K. Masuda, M. Kawabata, H. E. G. Powrie, "Advanced condition monitoring of tapered roller bearings, Part 1,” Tribology International, vol. 42, no. 11, pp. 1846-1856, 2009.

[8] T. J. Harvey, R. J. K. Wood, and H. E. G. Powrie, "Electrostatic wear monitoring of rolling element bearings,” Wear, vol. 263, no. 7, pp. 14921501, 2007.

[9] S. Morris, "Real-time electrostatic charge monitoring of the wear surfaces and debris generated by sliding bearing steel contacts," University of Southampton, 2003.

[10] T. J. Harvey, R. J. K. Wood, G. Denuault, H. E. G. Powrie, "Investigation of electrostatic charging mechanisms in oil lubricated tribo-contacts,” Tribology International, vol. 35, no. 9, pp. 605-614, 2002.

[11] J. B. Gajewski, "Electrostatic flow probe and measuring system calibration for solids mass flow rate measurement," Journal of Electrostatics, vol. 45, no 4, pp. 255-264, 1999.

[12] J. Q. Zhang and Y. Yan, "On-line continuous measurement of particle size using electrostatic sensors,” Powder Technology, vol. 135, pp. 164168, 2003.

[13] Y. Yan, B. Byrne, S. Woodhead and J. Coulthard, "Velocity measurement of pneumatically conveyed solids using electrodynamic sensors," Measurement Science and Technology, vol. 6, no. 5, pp. 515, 1995.

[14] J. B. Gajewski, “Static feature of an electrostatic flow probe,” Journal of Electrostatics, vol. 48, no. 1, pp. 49-64, 1999.

[15] R. C. Liu and H. F. Zuo, "Mathematical model, simulation, and experimental calibration of electrostatic wear-site sensor”. IEEE Sensors Journal, vol. 17, no. 8, pp. 2428-2438, 2017.

[16] R. C. Liu, H. F. Zuo, J. Z. Sun, and L. Wang, "Electrostatic monitoring of wind turbine gearbox on oil-lubricated system". Proceedings of the Institution of Mechanical Engineers, Part C: Journal of Mechanical Engineering Science, vol. 231, no. 19, pp. 3649-3664, 2017.

[17] R. C. Liu, H. F. Zuo, J. Z. Sun, and L. Wang, "Simulation of electrostatic oil line sensing and validation using experimental results”. Tribology International, vol. 105, pp. 15-26, 2017.

[18] J. Wu, X. Cui, G. Zheng, Y. M. Wang, L. M. Lee, and C. Yang, "Wide field-of-view microscope based on holographic focus grid illumination,” Optics Letters, vol. 35, no. 13, pp. 2188-2190, 2010.

[19] M. Laslandes, M. Salas, C. K. Hitzenberger, and M. Pircher, "Increasing the field of view of adaptive optics scanning laser ophthalmoscopy," Biomedical Optics Express, vol. 8, no. 11, pp. 4811-4826, 2017. 